\title{
Electronic properties of Cs+CO coadsorbed on the Ru(0001) surface
}

Cite as: J. Chem. Phys. 108, 774 (1998); https://doi.org/10.1063/1.475437

Submitted: 03 September 1997 . Accepted: 02 October 1997 . Published Online: 04 June 1998

S. Fichtner-Endruschat, V. De Renzi, A. Morgante, S. Schwegmann, H. Bludau, R. Schuster, A. Böttcher, and $\mathrm{H}$. Over

\section{ARTICLES YOU MAY BE INTERESTED IN}

Anomalous hydrogen adsorption sites found for the $\mathrm{c}(2 \times 2)-3 H$ phases formed on the $\operatorname{Re}(1010)$ and $\mathrm{Ru}(10 \uparrow 0)$ surfaces

The Journal of Chemical Physics 108, 8671 (1998); https://doi.org/10.1063/1.476296

\section{Lock-in Amplifiers up to $600 \mathrm{MHz}$}
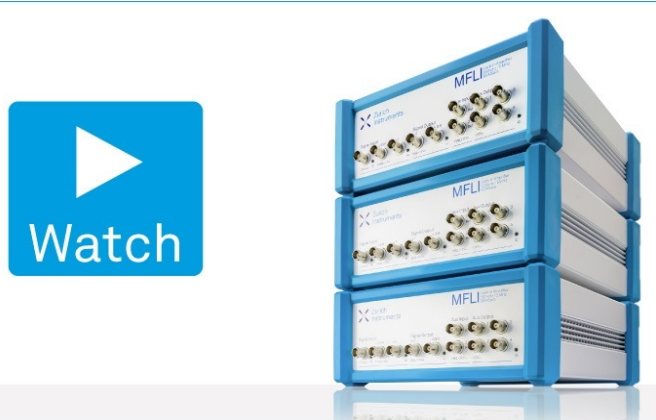

J. Chem. Phys. 108, 774 (1998); https://doi.org/10.1063/1.475437 


\section{Electronic properties of $\mathrm{Cs}+\mathrm{CO}$ coadsorbed on the $\mathrm{Ru}(0001)$ surface}

S. Fichtner-Endruschat and V. De Renzi

Fritz-Haber-Institut der Max-Planck-Gessellschaft, Faradayweg 4-6, D-14195 Berlin, Germany

A. Morgante

T.A.S.C.-I.N.F.M. Laboratory, Padriciano 99, I-34012 Trieste, Italy

S. Schwegmann, H. Bludau, and R. Schuster

Fritz-Haber-Institut der Max-Planck-Gessellschaft, Faradayweg 4-6, D-14195 Berlin, Germany

A. Böttcher

Humboldt-Universität zu Berlin, Institut für Physik, Oberflächenphysik und Atomstoßprozesse, Invalidenstrasse 110, D-10115 Berlin, Germany

H. Over ${ }^{\mathrm{a})}$

Fritz-Haber-Institut der Max-Planck-Gessellschaft, Faradayweg 4-6, D-14195 Berlin, Germany

(Received 3 September 1997; accepted 2 October 1997)

The variation of the Cs $6 s$ and the $\mathrm{Cs} 5 p$ emission in $\mathrm{He}^{*}$ and $\mathrm{Ne}^{*}$ metastable deexcitation spectroscopy (MDS) as a function of the $\mathrm{CO}$ exposure indicates a demetallization of the $\mathrm{Ru}(0001)$ $(2 \times 2)$-Cs and the $\mathrm{Ru}(0001)-(\sqrt{3} \times \sqrt{ } 3) R 30^{\circ}$-Cs surfaces upon $\mathrm{CO}$ coadsorption. This observation corroborates a (substrate-mediated) charge transfer from the Cs atom to the $2 \pi^{*}$ orbital of $\mathrm{CO}$. With the $\mathrm{Ru}(0001)-(2 \times 2)-\mathrm{Cs}$ system even at $\mathrm{CO}$ saturation, MD spectra show emission associated with the Cs $6 s$ state, indicating that the Cs atoms are not completely ionized. Exposing the $(\sqrt{ } 3$ $\times \sqrt{ } 3) R 30^{\circ}$-Cs-pre-covered $\mathrm{Ru}(0001)$ to $\mathrm{CO}$, surplus $\mathrm{Cs}$ of the first layer is displaced into a second layer. In this way, $\mathrm{CO}$ molecules are able to be accommodated into the first layer. Desorbing this second layer Cs by heating the sample to $600 \mathrm{~K}$ produces a $(2 \times 2)$ structure with one Cs and CO in the unit cell as evidenced by MDS and low energy electron diffraction. (c) 1998 American Institute of Physics. [S0021-9606(98)02902-X]

\section{INTRODUCTION}

The coadsorption of $\mathrm{CO}$ and alkali metals on transition metal surfaces has been envisioned as a workhorse to study the role of alkali metals as promoters in catalytic reactions, such as carbon monoxide hydrogenation and oxidation. ${ }^{1}$ Most of the work has been performed for $\mathrm{K}$ and $\mathrm{CO}$ coadsorbed on various metal surfaces concentrating on their electronic and vibrational properties. ${ }^{1,2}$ These studies were, however, based on ad hoc assumptions about the atomic geometry of this coadsorption system since this piece of information was not available at that time. ${ }^{3}$

Recently, the coadsorption of $\mathrm{CO}$ and $\mathrm{Cs}$ on $\mathrm{Ru}(0001)$ has been investigated, ${ }^{4,5}$ thereby providing detailed information on the atomic geometry and the vibrational properties. However, for this particular system the electronic properties are hardly known. Starting from a $(2 \times 2)$-Cs-pre-covered $\mathrm{Ru}(0001)$ surface, coadsorption of $\mathrm{CO}$ leads to an improved ordering and the formation of the mixed $\mathrm{Cs}+\mathrm{CO}$ overlayer. A corresponding low energy electron diffraction (LEED) structure analysis ${ }^{6}$ indicated that Cs atoms remain in on-top position (as also found for $\left.\mathrm{Ru}(0001)-(2 \times 2)-\mathrm{Cs}^{7}\right)$, while the $\mathrm{CO}$ molecule changes its adsorption site from on top, as found on the clean $\mathrm{Ru}(0001)$ surface, ${ }^{8}$ to the threefold hollow site in the mixed $\mathrm{Cs}+\mathrm{CO}$ overlayer. This site change was ascribed to a purely electronic effect. $\mathrm{CO}$ molecules can

\footnotetext{
a) Author to whom correspondence should be addressed. Electronic mail: over@fhi-berlin.mpg.de; Fax: ++49-30-84135106.
}

utilize the enhanced charge density at the surface due to the Cs overlayer by a (substrate-mediated) charge transfer into the $2 \pi^{*} \mathrm{CO}$ molecular orbital. Since back donation is more efficient in highly coordinated sites than in on-top positions, $\mathrm{CO}$ changes its adsorption site. This view of modified $\mathrm{CO}-\mathrm{Ru}$ bonding is corroborated by a recent high resolution electron energy loss spectroscopy (HREELS) study which indicated a lowering of the $\mathrm{C}-\mathrm{O}$ stretch vibrational frequency upon Cs coadsorption. ${ }^{4}$ In addition, this HREELS study indicated that the original "metallization" of the $\mathrm{Cs}(2$ $\times 2$ ) overlayer, i.e., the delocalization of the Cs $6 s$ electron wave function across the surface, is removed by coadsorbed $\mathrm{CO}$ molecules.

Starting instead with a full monolayer of Cs, i.e. a $(\sqrt{ } 3$ $\times \sqrt{ } 3) R 30^{\circ}$-Cs structure, CO uptake is slowed down by at least two orders of magnitude. Still, some CO can stick to the surface, eventually forming a bond with the Ru substrate and simultaneously forcing some of the Cs atoms to move from the first to the second layer. ${ }^{9}$ Altogether these findings demonstrate an intimate interrelation of geometric and vibrational properties with electronic properties which motivated us to study the $\mathrm{Cs}+\mathrm{CO}$ coadsorption system on $\mathrm{Ru}(0001)$ with spectroscopic methods such as metastable deexcitation spectroscopy (MDS) and ultraviolet photoelectron spectroscopy (UPS). The combination of UPS and MDS is particularly useful since it allows to discriminate between electronic properties merely of the outermost layer (MDS) and those of a slab consisting of several layers (UPS). Additionally, MDS is quite sensitive to $s$-like charge density, while UPS is not. 
On transition metal surfaces MDS allows the investigation of states close to the Fermi level which are created by the formation of the chemisorption bond. These states are usually masked in UPS by strong emission from the metallic $d$ states so that both techniques are complementary to some extent. Recent MDS measurements of $\mathrm{Li}$ and $\mathrm{Na}$ films on $\mathrm{Ru}(0001)$ have shown that the state of metallization ${ }^{10,11}$ can be readily monitored by MDS.

\section{EXPERIMENTAL DETAILS}

The measurements were conducted on the same $\mathrm{Ru}(0001)$ sample in different ultrahigh vacuum (UHV) chambers. One chamber ${ }^{5}$ contains a four-grid back-view LEED optics, auger electron spectroscopy (AES), thermal desorption spectroscopy (TDS), and facilities to clean and prepare the $\mathrm{Ru}(0001)$ surface. The sample temperature can be varied from $40 \mathrm{~K}$ (by cooling with liquid $\mathrm{He}$ ) to $1530 \mathrm{~K}$ (by direct resistive heating). The other experimental system ${ }^{12}$ consists of an atomic beam source in which metastable noble gas atoms (either $\mathrm{He}$ or $\mathrm{Ne}$ ) are produced by electron impact. The atomic beam source is connected with the spectrometer chamber equipped with standard facilities for sample preparation, a He discharge lamp for UPS, and a hemispherical electron energy analyzer for recording the kinetic energy distribution of electrons emitted from the surface. The metastable atoms are deexcited upon colliding with the sample surface which causes the emission of electrons from the outermost layer of the surface. For the kinetic energy of the emitted electrons an energy balance analogous to UPS holds. The use of $\mathrm{He}$ or $\mathrm{Ne}$ metastable atoms allows to preferentially probe orbitals either with $\sigma$ symmetry or with $\pi$ symmetry, respectively. ${ }^{13(b)}$ Since the work function of the sample surface was below $3 \mathrm{eV}$ in all of our measurements, the major deexcitation mechanism of the metastable noble gas atoms is Penning ionization, i.e., an electron transition from states of the mixed $\mathrm{Cs}+\mathrm{CO}$ overlayer into the ground state hole of the impinging metastable atom is accompanied by the emission of the electron from the metastable state orbital. $^{13(a)}$ This electron carries the energy difference between the excitation energy of the $\mathrm{He}^{*}$ and the binding energy of the electron from the surface and, hence, directly probes the density of states of the outermost surface layer.

Cesium was evaporated from well outgassed dispenser sources (SAES Getter, Inc.). At a sample temperature of 220 $\mathrm{K}$ the deposition rate was about $1 \mathrm{ML} / \mathrm{min}$. Specific submonolayer coverages were prepared by first evaporating an alkali metal layer of approximately $2 \mathrm{ML}$, followed by flash annealing to predetermined temperatures to desorb excess alkali metal. The $(\sqrt{ } 3 \times \sqrt{ } 3) R 30^{\circ}-\mathrm{Cs}$ and the $(2 \times 2)$-Cs overlayers need annealing to temperatures of 332 and $523 \mathrm{~K}$, respectively (heating rate $5 \mathrm{~K} / \mathrm{s})$. CO $(99.97 \%)$ was dosed by backfilling the chamber with a partial pressure of 1 $\times 10^{-8}$ mbar.
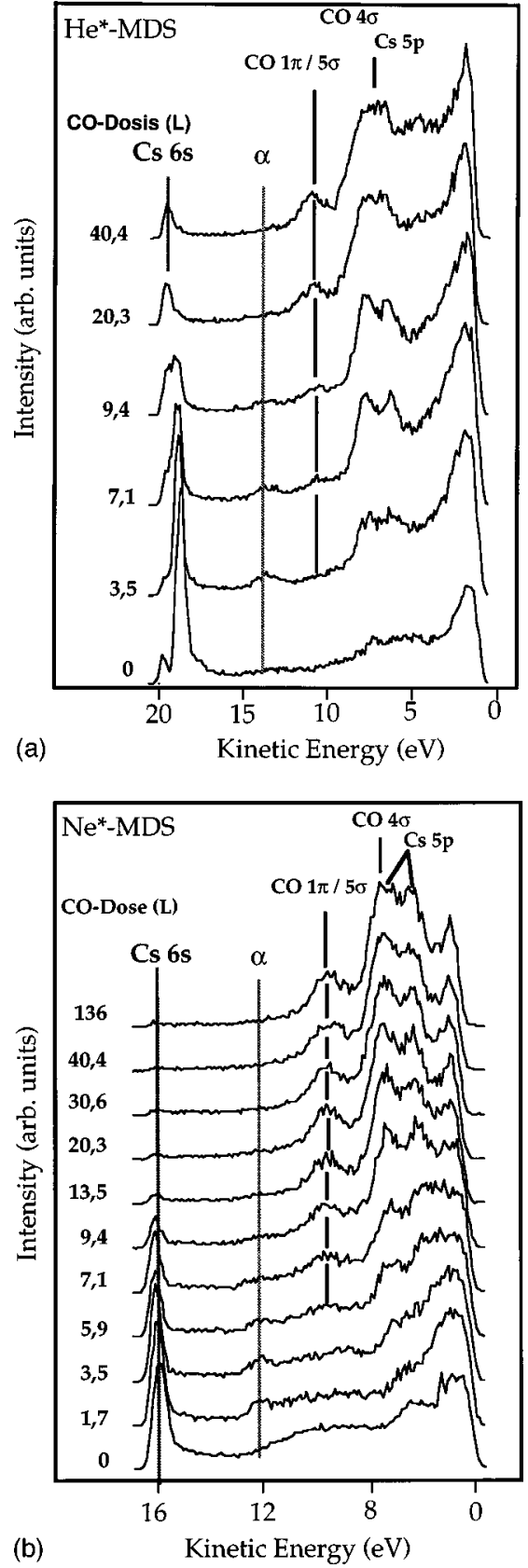

FIG. 1. (a) $\mathrm{He}^{*} \mathrm{MD}$ spectra and (b) $\mathrm{Ne}^{*} \mathrm{MD}$ spectra from a $(2 \times 2)$-Cs-precovered $\mathrm{Ru}(0001)$ surface which is exposed to various amounts of $\mathrm{CO}$ as specified in the figure.

\section{RESULTS AND DISCUSSION}

\section{A. CO adsorption on the $(2 \times 2)$-Cs-precovered $\mathrm{Ru}(001)$ surface}

Figure 1 shows $\mathrm{He}^{*} \mathrm{MD}$ spectra from a $(2 \times 2)$-Cs-precovered $\mathrm{Ru}(0001)$ surface which is exposed to increasing amounts of CO. The electron emission is shown as a function of the kinetic energy. A representation in terms of binding energies would require the knowledge of the effective excitation energy of the impinging metastable $\mathrm{He}$ or $\mathrm{Ne}$ atoms which is in general influenced by the chemical composition of the outermost surface. For metal surfaces this leads to variations of $E^{*}$ of several $100 \mathrm{meV} .{ }^{13}$ As discussed in detail 


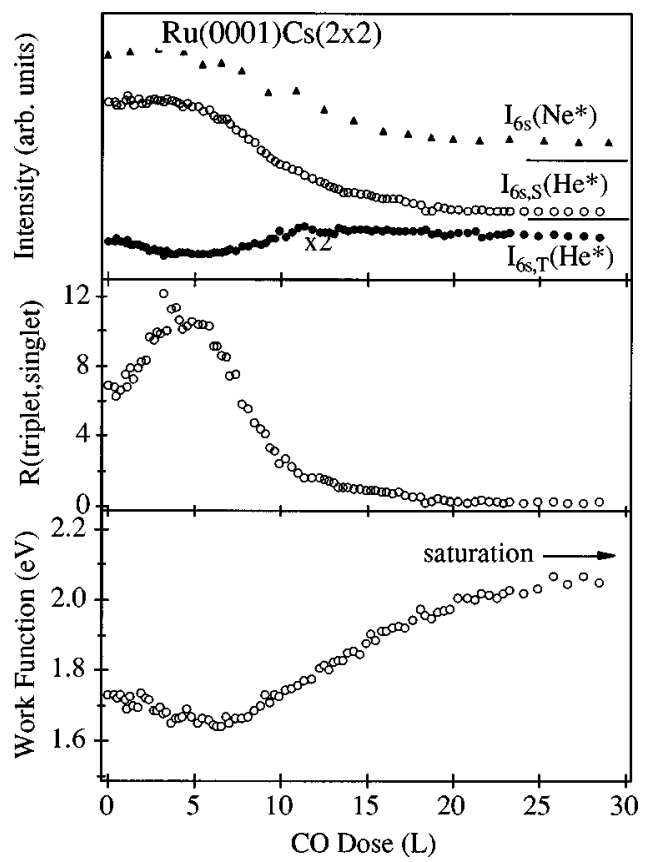

FIG. 2. The intensity of the Cs $6 s$-derived peaks in $\mathrm{He}^{*}$ and $\mathrm{Ne}^{*}$ MDS, singlet-triplet conversion rate $R$, and the work function change during $\mathrm{CO}$ exposure of the $(2 \times 2)$-Cs-pre-covered $\mathrm{Ru}(0001)$ surface.

in Ref. 10 the emission of electrons with highest kinetic energy originates from the Cs $6 s$ level excited by either singlet $(20.3 \mathrm{eV})$ or triplet $(19.1 \mathrm{eV}) \mathrm{He}^{*}$ atoms. Since the majority $(\geqslant 90 \%)$ of impinging metastable $\mathrm{He}^{*}$ atoms are originally in the singlet state $\left[\mathrm{He}^{*}\left(2^{1} S\right)\right]$, and the MD spectra show a strong $\mathrm{Cs} 6 s$ emission at a kinetic energy related to the $\mathrm{He}$ triplet state, an efficient singlet-triplet conversion has taken place prior to the actual deexcitation process reflecting the presence of a rather metallic Cs overlayer. ${ }^{11}$ As summarized in Fig. 2, with progressing $\mathrm{CO}$ exposure, the total emission from the Cs $6 s$ level decreases monotonically, whereas the singlet-triplet conversion rate $R$ (defined as the ratio of singlet to triplet peak intensity in MDS) increases first before it decreases steeply beyond $9 \mathrm{~L}$. The maximum of $R$ is related to the minimum of the work function change $\Delta \Phi$; the work function change was determined by the width of the UPS spectra. The decrease of $R$ is consistent with a gradual "demetallization" 10 of the surface due to $\mathrm{CO}$ adsorption as also observed with HREELS. ${ }^{4}$ Regardless of the specific mechanisms suggested in the literature, ${ }^{11,14}$ the singlettriplet conversion step prior to the final Penning ionization step requires both occupied and unoccupied states near the Fermi level, i.e., a metallic surface. It is remarkable that, even for high $\mathrm{CO}$ exposures, a $6 s$-derived MD signal is discernible which implies that the Cs atoms are still not completely ionized.

Electron emission with smaller kinetic energy (He* MDS) is assigned to $5 \sigma+1 \pi$ of $\mathrm{CO}(\approx 10 \mathrm{eV})$ and to the Cs $5 p$-derived states $(\approx 8 \mathrm{eV})$. The assignment was made by comparison with the corresponding UP spectrum for which energy levels are known from the literature. ${ }^{15,16}$ As expected, the intensity of the $5 \sigma+1 \pi$-derived peak at about $10 \mathrm{eV}$

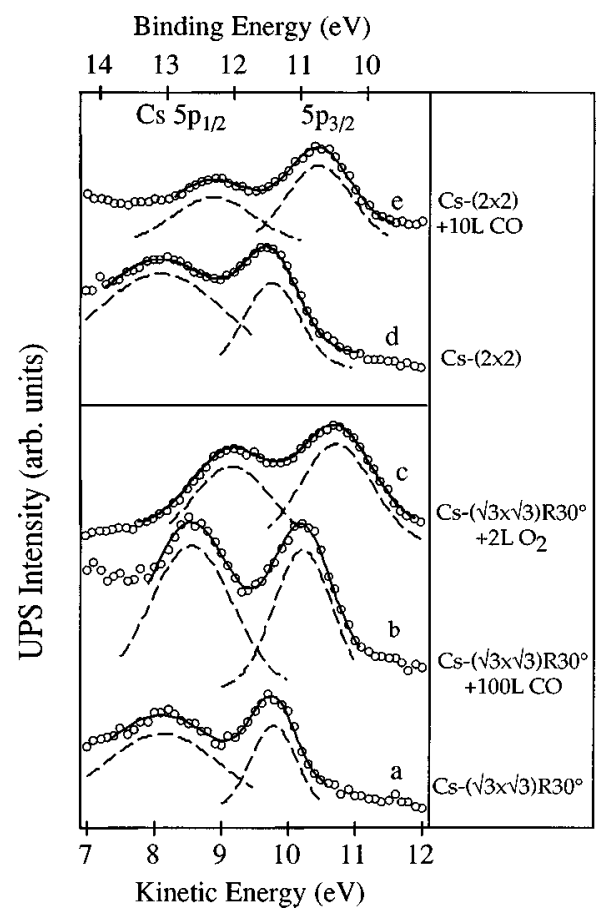

FIG. 3. UP spectra in the energy region of the Cs $5 p$ doublet for various overlayers on $\mathrm{Ru}(0001): \quad$ (a) $(\sqrt{3} \times \sqrt{ } 3) R 30^{\circ}-\mathrm{Cs}$ clean, (b) $(\sqrt{3}$ $\times \sqrt{3}) R 30^{\circ}-\mathrm{Cs}+100 \mathrm{~L} \mathrm{CO},(\mathrm{c})(\sqrt{3} \times \sqrt{ } 3) R 30^{\circ}-\mathrm{Cs}+2 \mathrm{~L} \mathrm{O}_{2}$, (d) $(2 \times 2)-\mathrm{Cs}$ clean, (e) $(2 \times 2)-\mathrm{Cs}+1 \mathrm{O}$ L CO.

increases gradually with $\mathrm{CO}$ exposure [cf. Fig. 1(a)]. The electron emission from the $4 \sigma$ CO state overlaps with the signal originating from the Cs $5 p$ states and is therefore obscured.

The Cs $5 p$ doublet shifts to lower binding energies upon CO adsorption as evidenced by UPS (Fig. 3(d) and 3(e)); a similar effect was also observed for oxygen adsorption on a Cs-pre-covered $\mathrm{Ru}(0001)$ surface. ${ }^{17}$ Intuitively one would expect to find a shift of the Cs $5 p$ levels to larger binding energies since the transfer of Cs $6 s$ charge density to the $\mathrm{CO}$ molecules should lead to a reduction of the screening charge density in the core region, thus increasing the binding energies of the Cs $5 p$ core levels. On the other hand, it is frequently observed that a core level shifts to lower binding energy when the coordination of the respective species is increased, i.e., when the adsorbate atoms are less exposed to the vacuum. ${ }^{18}$

The negative chemical shift of the Cs $5 p$ levels can alternatively be explained by a charge redistribution between $6 s$ and $5 d$ levels of $\mathrm{Cs}$, as first proposed for $\mathrm{BaO}^{19}$ and later modified for the case of Cs oxides. ${ }^{20}$ According to this model, Cs loses $6 s$ charge density but concomitantly receives $5 d$ charge density. This effect is a consequence of the surface compression of the Cs overlayer due to the bond formation of $\mathrm{Cs}$ with the topmost $\mathrm{Ru}$ layer; recall that a $6 s \rightarrow 5 d$ charge transfer is also found for Cs bulk material which is subject to high external pressures. ${ }^{21}$ As a net result, the Cs $6 s$ becomes more strongly polarized towards the $\mathrm{Ru}(0001)$ substrate. The population of $5 d$ states, however, causes the Cs $5 p$ levels in UPS and MDS to shift to lower binding energies. Coadsorbing $\mathrm{CO}$ molecules squeeze into 
the Cs overlayer which compresses the Cs charge density in the $(2 \times 2)-C s$ overlayer, thus enforcing the $6 s 5 d$ hybridization. This might result in the observed shift of the Cs $5 p$ levels in UPS and MDS. In other words, coadsorbing CO molecules even further polarize the Cs $6 s$ state toward the $\mathrm{Ru}$ substrate. The stronger $\mathrm{Cs} 6 s$ polarization toward $\mathrm{Ru}$ is also supported by the evolution of the Cs $5 p$ emission in $\mathrm{He}^{*}$ MDS [cf. Fig. 1(a)] which increases markedly with CO exposure. Obviously, the coadsorbed $\mathrm{CO}$ molecules consume the (delocalized) Cs $6 s$ charge density in the overlayer which in turn leads to an improved exposure of Cs $5 p$ orbitals to the impinging metastable $\mathrm{He}^{*}$ and $\mathrm{Ne}^{*}$ atoms.

Similar to the case of $\mathrm{K}+\mathrm{CO}$ on $\mathrm{Ru}(0001),{ }^{15}$ but different from the case of $\mathrm{K}+\mathrm{CO}$ on $\mathrm{Ni}(111),{ }^{22}$ no $2 \pi^{*}$-derived molecular orbital was seen in the MD spectra for the Cs-precovered $\mathrm{Ru}(0001)$ surface. The MD spectra (Fig. 1) do reveal intense electron emission from the $5 \sigma+1 \pi$ of CO. Recalling the particular shapes of the $5 \sigma$ and $1 \pi$ molecular orbitals, ${ }^{23}$ the $1 \pi$ orbital reaches farther out into the vacuum region than the $5 \sigma$ orbital. Hence, it is plausible to assume that the $\mathrm{CO}(5 \sigma+1 \pi)$ emission consists mainly of electrons ejected from the $1 \pi$ orbital, as also concluded by Jänsch et al. ${ }^{24}$ The $\mathrm{Ne}^{*}$ MD spectra of the same system [shown in Fig. 1(b)] are quite similar to those spectra taken with $\mathrm{He}^{*}$ atoms [cf. Fig. 1(a)]. The main difference is the improved resolution of the Cs $5 p$ levels in the $\mathrm{Ne}$ MD spectrum since $\mathrm{Ne}^{*}$ is expected to be more sensitive to orbitals with $\pi$ symmetry than $\mathrm{He}^{*}$. An additional feature, which becomes more obvious in $\mathrm{Ne}^{*}$ MD spectra than in corresponding $\mathrm{He}^{*} \mathrm{MD}$ spectra, is the narrowing of the Cs $5 p_{1 / 2}$ linewidth upon coadsorption of $\mathrm{CO}$, the $\mathrm{Cs} 5 p_{3 / 2}$ linewidth remains constant.

In Fig. 4 we compare the spectra of $\mathrm{He}^{*}$ and $\mathrm{Ne}^{*} \mathrm{MDS}$ with corresponding UPS measurements. There is a one-toone correspondence of peaks in the $\mathrm{Ne}^{*} \mathrm{MD}$ spectrum with those in the UP spectrum but in the $\mathrm{He}^{*}$ MD spectrum the $\mathrm{Cs}$ $5 p$ and $\mathrm{CO}(5 \sigma+1 \pi)$ associated peaks are shifted by $0.8 \mathrm{eV}$ to higher binding energies. As this shift is similar to the energy difference between singlet and triplet metastable He atoms, one might interpret the $\mathrm{Cs} 5 p$ and $\mathrm{CO}(5 \sigma+1 \pi)$ emissions in the He MD spectra as due to quenching of triplet $\mathrm{He}$ atoms.

Next, we concentrate on the region between 10 and $5 \mathrm{eV}$ kinetic energy in MD and UP spectra where a $\mathrm{CO}$-alkali metal hybrid state is discussed for $\mathrm{CO} / \mathrm{K} / \mathrm{Ru}(0001)^{15}$ and called ' $\alpha$ ' peak (cf. Fig. 1). A feature at $12 \mathrm{eV}$ emerges in $\mathrm{Ne}^{*} \mathrm{MDS}$ (and at $14 \mathrm{eV}$ in $\mathrm{He}^{*} \mathrm{MDS}$ ) with ongoing $\mathrm{CO}$ deposition and disappears finally beyond $10 \mathrm{~L}$. This COderived peak $(\alpha)$ is not present in the pure $\mathrm{CO}$ spectrum, and therefore it might be attributed to the intense $\mathrm{Cs}-\mathrm{CO}$ interaction. It was carefully checked that neither oxygen impurity in the $\mathrm{CO}$ gas and $\mathrm{CO}$ dissociation nor background gas adsorption during the experiment were responsible for these emissions. MDS is sensitive to orbitals which are polarized toward the vacuum region and to orbitals with $\sigma$ symmetry. Therefore, considering the high intensity in MDS, the $\alpha$ state is interpreted to be polarized toward the vacuum. A comparison of the evolution of $\alpha$ with those of the singlet-triplet conversion rate $R$ and the intensity of Cs $6 s$ reveals a close
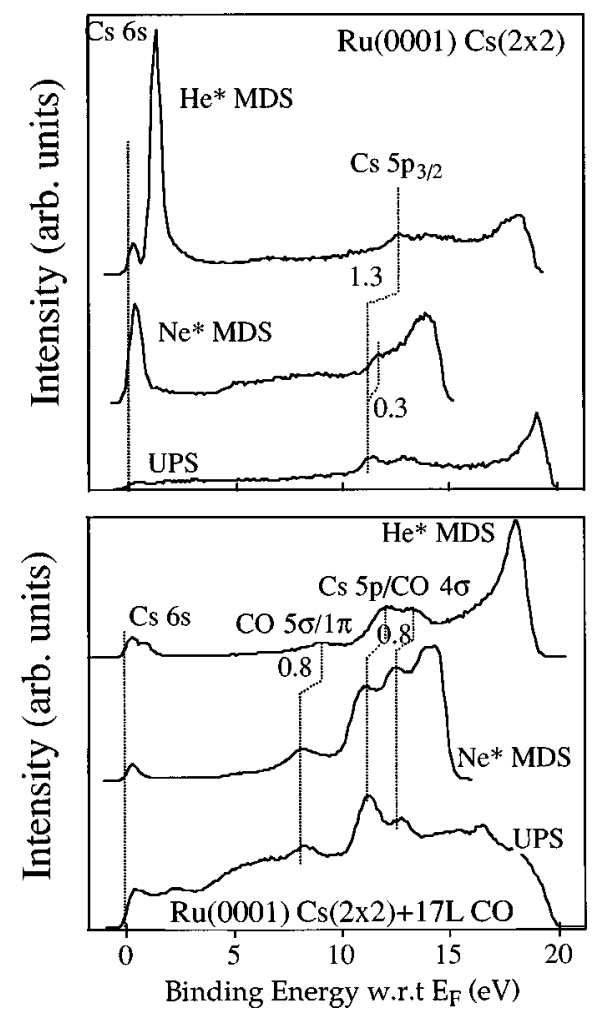

FIG. 4. Comparison of $\mathrm{He}^{*}$ and $\mathrm{Ne}^{*} \mathrm{MD}$ spectra with corresponding UP spectra for (a) $\mathrm{Ru}(0001)-(2 \times 2)-\mathrm{Cs}$ and (b) $\mathrm{Ru}(0001)-(2 \times 2)-\mathrm{Cs}+17 \mathrm{~L} \mathrm{CO}$.

relation between $\alpha$ and the demetallization of the surface. The interaction of $\mathrm{CO}$ with this delocalized $\mathrm{Cs} 6 s$ charge density leads to the electron emission $\alpha$. With prolonged $\mathrm{CO}$ exposure the delocalized Cs $6 s$ density diminishes with the consequence that $\alpha$ also disappears in MDS. Hence, one might argue that the $\alpha$ peak represents a direct interaction of delocalized Cs $6 s$ charge density with the CO molecule.

In Fig. 3 we compare the Cs $5 p$-associated features in UPS of the Cs-pre-covered $\mathrm{Ru}(0001)$ surfaces with those of the $\mathrm{Cs}+\mathrm{CO}$ and $\mathrm{Cs}+\mathrm{O}$-covered $\mathrm{Ru}(0001)$ surfaces. The most remarkable variation in these spectra is the linewidth of the Cs $5 p_{1 / 2}$ level. Upon $\mathrm{CO}$ exposure, the Cs $5 p_{1 / 2}$ profile narrows quite substantially. Without $\mathrm{CO}$, the emission from the Cs $5 p_{1 / 2}$ level is quite broad since the core hole is filled rapidly by an electron from the $\mathrm{Cs} 5 p_{3 / 2}$ which leads to electron emission from the Cs $6 s$ band via an Auger-like transition. The direct Cs $5 p_{1 / 2}$ - Cs $5 p_{3 / 2}$ transition is dipole forbidden. Therefore, the presence of Cs $6 s$ charge density close to the Fermi level is mandatory for this so-called CosterKronig transition to be efficient. The emitted electrons have very low kinetic energy and are usually obscured by the secondary electron emission in UPS. If we now add $\mathrm{CO}$ and $\mathrm{O}$ (both are electronegative species) to the Cs overlayer, the Cs $6 s$ charge density is gradually depleted through a charge transfer from $\mathrm{Cs}$ to $\mathrm{CO}$ or $\mathrm{O}$. This process reduces the probability for a Coster-Kronig transition and therefore increases the lifetime of the Cs $5 p_{1 / 2}$ hole which in turn narrows the Cs $5 p_{1 / 2}$ profile as evidenced in UPS. 

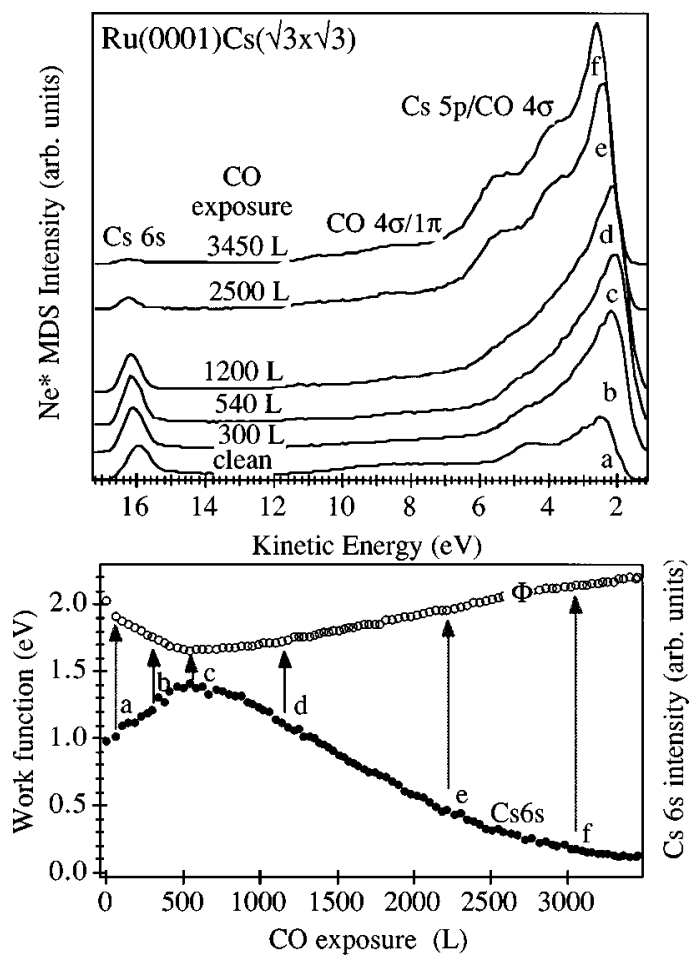

FIG. 5. (a) $\mathrm{Ne}^{*} \mathrm{MD}$ spectra from a $(\sqrt{3} \times \sqrt{3}) R 30^{\circ}$-Cs-pre-covered $\mathrm{Ru}(0001)$ surface which is exposed to increasing amounts of $\mathrm{CO}$ as specified in the figure. (b) The intensity of the Cs $6 s$ emission in comparison with the work function change.

\section{B. CO adsorption on the $(\sqrt{ } 3 \times \sqrt{ } 3) R 30^{\circ}$-Cs-pre-covered $\mathrm{Ru}(0001)$ surface}

The Ne MD spectra of $\mathrm{Ru}(0001)-(\sqrt{ } 3 \times \sqrt{ } 3) R 30^{\circ}$-Cs exposed to increasing doses of $\mathrm{CO}$ are summarized in Fig. 5. The Cs $6 s$-derived MD signal I $(6 s)$ first increases until the work function has passed the minimum and then decreases at higher doses [Fig. 6(b)]; note that the singlet-triplet splitting is too small to be resolved for metastable $\mathrm{Ne}$ atoms. The behavior of the $6 s$ emission is in contrast to that observed for the $(2 \times 2)$-Cs-pre-covered surface for which this emission in the $\mathrm{Ne}^{*} \mathrm{MD}$ spectra steadily decreases with $\mathrm{CO}$ exposure. Additionally, on the $(\sqrt{3} \times \sqrt{ } 3) R 30^{\circ}$-Cs surface the sticking coefficient is two orders of magnitude smaller than for the $(2 \times 2)$-Cs. Hence, the demetallization of the $(\sqrt{ } 3$ $\times \sqrt{ } 3) R 30^{\circ}$-Cs surface needs much higher $\mathrm{CO}$ doses [cf. Fig. $5(\mathrm{~b})]$.

He MD spectra from the $(\sqrt{ } 3 \times \sqrt{ } 3) R 30^{\circ}$-Cs-pre-covered $\mathrm{Ru}(0001)$ surface exposed to $200 \mathrm{~L} \mathrm{CO}$ (cf. Fig. 6) reveal a surprisingly high Cs $6 s$-derived signal. Concomitant LEED measurements of this surface show a complex LEED pattern with dominating spots at positions of a rotated $(2 \times 2)$ structure. A similar LEED pattern was also reported by Kondoh and Nozoye. ${ }^{9}$ Upon heating this overlayer to $600 \mathrm{~K}$, the MD spectrum transforms into a spectrum which is identical to that from the $(2 \times 2)-\mathrm{Cs}+\mathrm{CO}$ surface prepared at room temperature. From TDS measurements, on the other hand, it is found that part of the Cs desorbs during this annealing process (while all CO molecules stay on the surface), leaving a well-ordered $(2 \times 2)$ structure behind as indicated by the

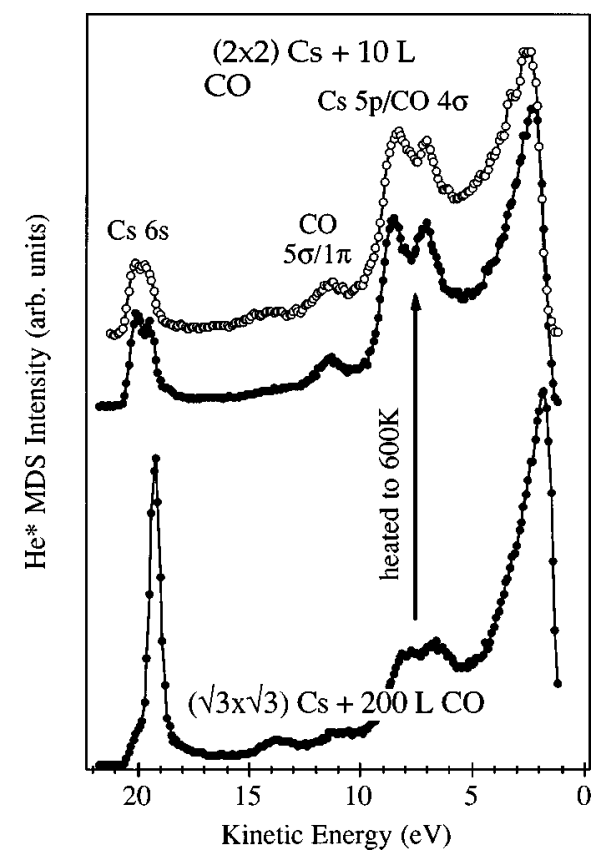

FIG. 6. He* MD spectra from the $(\sqrt{3} \times \sqrt{ } 3) R 30^{\circ}$-Cs-pre-covered $\mathrm{Ru}(0001)$ surface exposed to $200 \mathrm{~L} \mathrm{CO}$ and after annealing to $600 \mathrm{~K}$ in comparison with the $\mathrm{He}^{*} \mathrm{MD}$ spectrum of the $\mathrm{Ru}(0001)-(2 \times 2)-\mathrm{Cs}+10 \mathrm{~L} \mathrm{CO}$.

LEED pattern. LEED $I-V$ curves taken for this a priori unknown $(2 \times 2)$ structure are almost identical to those found for the $(2 \times 2)-\mathrm{Cs}+1 \mathrm{CO}$ phase but significantly different from LEED $I-V$ curves of the $(2 \times 2)-\mathrm{Cs}+2 \mathrm{CO}$ phase. $^{5}$ Therefore, this $(2 \times 2)$ phase can safely be ascribed to a $(2$ $\times 2)$-Cs $+1 \mathrm{CO}$ overlayer. The question left is where the excess Cs was located prior to desorption via heating to $600 \mathrm{~K}$ : Either the Cs atoms were placed on top of the mixed $(2 \times 2)$ $\mathrm{Cs}+1 \mathrm{CO}$ layer, or they could have been embedded in the $\mathrm{Cs}+1 \mathrm{CO}$ layer. The strong $\mathrm{Cs} 6 \mathrm{~s}$ emission in MDS after CO exposure, however, evidences the formation of a second $\mathrm{Cs}$ layer on top of the $\mathrm{Cs}+1 \mathrm{CO}$ layer.

The finding of second layer $\mathrm{Cs}$ is also consistent with the experimentally observed variation of the emission of Cs $5 p$ and the $5 \sigma+1 \pi$-CO which are both significantly larger after than before heating the sample to $600 \mathrm{~K}$. With Cs in the second layer, these features are substantially damped, while after removing the second layer Cs atoms, the Cs $5 p$ and also the $\mathrm{CO}-(5 \sigma+1 \pi)$-derived emissions are appreciably enhanced. Altogether these findings confirm the formation of second layer $\mathrm{Cs}$ atoms upon adsorption of $\mathrm{CO}$ onto the $(\sqrt{ } 3$ $\times \sqrt{ } 3) R 30^{\circ}$-Cs surface, as has already been proposed by Kondoh and Nozoye. ${ }^{9}$

The above picture is comprehensive only for $\mathrm{CO}$ doses up to $1000 \mathrm{~L}$. When exposing the $(\sqrt{3} \times \sqrt{ } 3) R 30^{\circ}$-Cs surface to even higher $\mathrm{CO}$ doses $(3500 \mathrm{~L})$, some drastic changes occurred in thermal desorption spectra (cf. Fig. 7). The thermal desorption of $\mathrm{CO}, \mathrm{Cs}$, and $\mathrm{CO}_{2}$ was monitored simultaneously. While for the case of $1000 \mathrm{~L} \mathrm{CO}$. [cf. Fig. 7(a)], only very few $\mathrm{CO}_{2}$ could be detected; the $\mathrm{CO}_{2}$ signal increases by a factor of 8 when dosing $3500 \mathrm{~L}$ CO instead [cf. Fig. 7(b)]. Since the CO exposure is increased only by a 


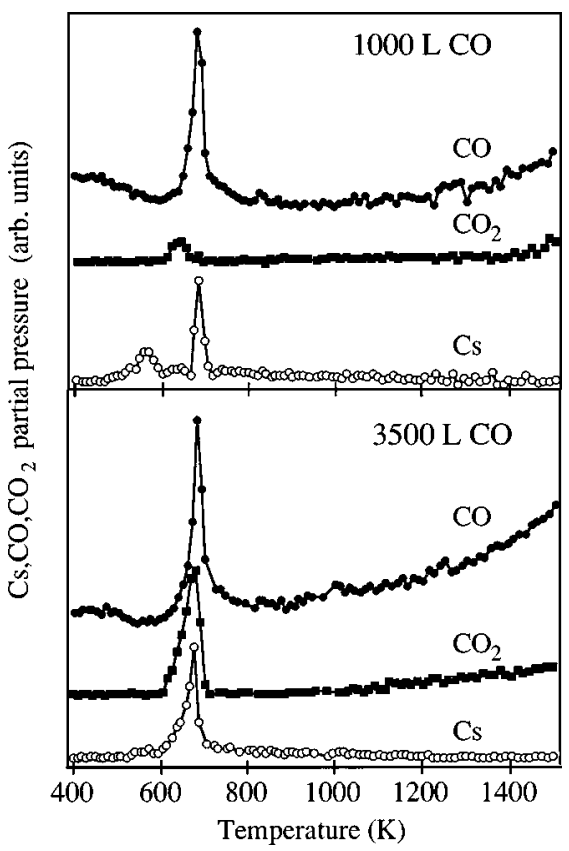

FIG. 7. Thermal desorption spectra of $\mathrm{Cs}, \mathrm{CO}$, and $\mathrm{CO}_{2}$. The $\mathrm{Ru}(0001)-(\sqrt{3} \times \sqrt{3}) R 30^{\circ}$-Cs surface is exposed to (a) $1000 \mathrm{~L} \mathrm{CO}$ (b) 3500 L CO.

factor of 3.5 at fixed $\mathrm{CO}$ partial pressure, this effect cannot be simply related to $\mathrm{CO}_{2}$ stemming from the residual gas or from the impurity concentration of the admitted $\mathrm{CO}$ gas. Parallel with this increase in $\mathrm{CO}_{2}$ production, the $\mathrm{Cs} \mathrm{TD}$ spectrum also changed. The weaker bound $\mathrm{Cs}$, which is attributed to second layer Cs, disappears for the case of $3500 \mathrm{~L} \mathrm{CO}$, and instead the co-desorption peak broadens and shifts to lower temperatures. The LEED patterns are also quite different. While for the $1000 \mathrm{~L} \mathrm{CO}$ case the above mentioned complex rotated $(2 \times 2)$ LEED pattern is observed, the LEED pattern for the $3500 \mathrm{~L} \mathrm{CO}$ case exhibits only simple $(2 \times 2)$ symmetry. Accompanying MDS measurements still show small emission due to the $\mathrm{Cs} 6 s$ for $1000 \mathrm{~L} \mathrm{CO}$, but this signal is largely suppressed upon CO exposure of $3500 \mathrm{~L}$. One may speculate that excessive $\mathrm{CO}$ exposure also turns the second layer Cs atoms into a more or less ionized species, which is involved in the production of $\mathrm{CO}_{2}$, or second layer Cs atoms simply desorb.

\section{CONCLUSIONS}

The variation of the electron emission in MDS due to the Cs $6 s$ level as a function of the $\mathrm{CO}$ exposure indicates a demetallization of the $\operatorname{Cs}(2 \times 2)$ and the $(\sqrt{ } \times \sqrt{ } 3) R 30^{\circ}$ - Cs overlayer upon $\mathrm{CO}$ coadsorption. This observation is in line with the interpretation of recent HREELS measurements ${ }^{4}$ and corroborates the idea of a pronounced (substratemediated) charge transfer from the Cs atom to the $2 \pi^{*}$ orbital of CO. Even at $\mathrm{CO}$ saturation, the $\mathrm{Cs}$ atoms are not completely ionized in the $(2 \times 2)$-Cs overlayer, as MD spectra still show an emission associated with Cs $6 s$ level. The $\mathrm{Cs}-\mathrm{CO}$ interaction manifests itself in the presence of a Cs-CO hybrid orbital $(\alpha)$ in MDS, which is polarized to- ward the vacuum and disappears beyond a $\mathrm{CO}$ exposure of $10 \mathrm{~L}$. One might argue that the $\alpha$ peak is related to a direct interaction of delocalized $\mathrm{Cs} 6 s$ charge density with $\mathrm{CO}$. Beyond a $\mathrm{CO}$ exposure of $10 \mathrm{~L}$ this interaction is suppressed due to demetallization.

The high-density $(\sqrt{ } 3 \times \sqrt{ } 3) R 30^{\circ}$-Cs-pre-covered $\mathrm{Ru}(0001)$ surface reveals some additional features. Upon adsorption of $\mathrm{CO}$, part of the $\mathrm{Cs}$ in the first layer is displaced into a second layer which enables the $\mathrm{CO}$ molecules to accommodate into the first layer. Removing this second layer Cs by heating the sample to $600 \mathrm{~K}$ produces a surface which is identical to the $(2 \times 2)-\mathrm{Cs}+1 \mathrm{CO}$ phase as evidenced by MDS and LEED. However, if the $(\sqrt{ } 3 \times \sqrt{ } 3) R 30^{\circ}$-Cs surface is exposed to even higher doses of CO (e.g., $3500 \mathrm{~L}$ ), the Cs $6 s$ signal in MDS diminishes, and simultaneously a species is created that leaves the surface as $\mathrm{CO}_{2}$, as evidenced by TDS measurements.

\section{ACKNOWLEDGMENTS}

We appreciate very much the valuable discussions with J. N. Andersen, K. Jacobi, and G. Ertl. V.D. acknowledges partial financial support from A. Della Riccia Foundation.

${ }^{1}$ G. Pirug and H. P. Bonzel, in The Chemical Physics of Solid Surfaces, Coadsorption, Promoters, and Poisons, edited by D. A. King and D. P. Woodruff (Elsevier, Amsterdam, 1993), Vol. 6, pp. 51-112.

${ }^{2}$ Physics and Chemistry of Alkali Adsorption, edited by H. P. Bonzel, A. M. Bradshaw, and G. Ertl (Elsevier, Amsterdam, 1989).

${ }^{3}$ R. D. Diehl and R. McGrath, Surf. Sci. Rep. 23, 43 (1996).

${ }^{4}$ K. Jacobi, H. Shi, M. Gruyters, and G. Ertl, Phys. Rev. B 49, 5733 (1994).

${ }^{5}$ H. Over, H. Bludau, R. Kose, and G. Ertl, Surf. Sci. 331-333, 62 (1995).

${ }^{6}$ H. Over, H. Bludau, R. Kose, and G. Ertl, Phys. Rev. B 51, 4661 (1995).

${ }^{7}$ H. Over, H. Bludau, M. Skottke-Klein, G. Ertl, W. Moritz, and C. T. Campbell, Phys. Rev. B 45, 8638 (1992).

${ }^{8}$ G. Michalk, W. Moritz, H. Pfnür, and D. Menzel, Surf. Sci. 129, 92 (1983).

${ }^{9}$ H. Kondoh and H. Nozoye, Surf. Sci. 334, 39 (1995).

${ }^{10}$ A. Böttcher, A. Morgante, R. Grobecker, T. Greber, and G. Ertl, Phys. Rev. B 49, 10607 (1994).

${ }^{11}$ B. Woratschek, W. Sesselmann, J. Küppers, G. Ertl, and H. Haberland, Phys. Rev. Lett. 55, 611 (1985).

${ }^{12}$ H. Conrad, G. Ertl, J. Küppers, W. Sesselmann, and H. Haberland, Surf. Sci. 121, 161 (1982).

${ }^{13}$ (a) W. Sesselmann, B. Woratschek, G. Ertl, J. Küppers, and H. Haberland, Surf. Sci. 146, 14 (1984); (b) G. Ertl and J. Küppers, Low Energy Electron and Surface Chemistry (VCH, Weinheim, 1985).

${ }^{14}$ R. Hemmen and H. Conrad, Phys. Rev. Lett. 67, 1314 (1991).

${ }^{15}$ G. H. Rocker, C. Huang, C. L. Cobb, H. Metiu, and R. M. Martin, Surf. Sci. 244, 103 (1991).

${ }^{16}$ T. K. Sham and J. Hrebek, J. Chem. Phys. 89, 1188 (1988).

${ }^{17}$ G. Ebbinghaus, W. Braun, A. Simon, and K. Berresheim, Phys. Rev. Lett. 37, 1779 (1976)

${ }^{18}$ W. F. Egelhoff, Jr., Surf. Sci. Rep. 6, 253 (1987); A. Nilsson, B. Eriksson, N. Mårtensson, J. N. Andersen, and J. Onsgard, Phys. Rev. B 38, 10357 (1988).

${ }^{19}$ G. K. Wertheim, J. Electron Spectrosc. Relat. Phenom. 34, 309 (1984).

${ }^{20}$ J. A. Rodriguez, J. Hrebek, M. Kuhn, and T. K. Sham, J. Phys. Chem. 97, 4737 (1993).

${ }^{21}$ M. Ross and A. K. McMahan, Phys. Rev. B 26, 4088 (1982).

${ }^{22}$ J. Lee, C. P. Hanrahan, J. Arias, R. M. Martin, and H. Metiu, Phys. Rev. Lett. 51, 1803 (1983).

${ }^{23}$ J. C. Campuzano, in Chemical Physics of Solid Surfaces and Heterogeneous Catalysis, edited by D. A. King and D. P. Woodruff (NorthHolland, Amsterdam, 1990), Vol. 3(a).

${ }^{24}$ H. J. Jänsch, C. Huang, A. Ludviksson, J. D. Redding, H. Metiu, and R. M. Martin, Surf. Sci. 222, 199 (1989). 